JOURNAL OF

SYNCHROTRON

RADIATION

ISSN 1600-5775

Received 26 July 2021

Accepted 11 August 2021

₹ Current address: Department of Chemistry, University of Manchester, Manchester M13 9PL, UK.

Keywords: extremely bright SR sources; materials science; macromolecular crystallography.

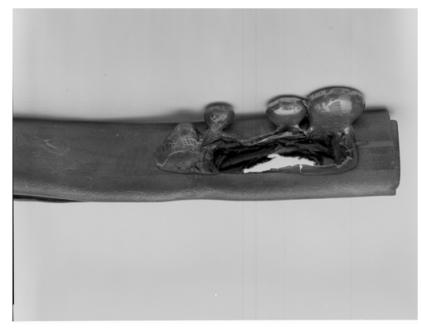

\section{Respect the synchrotron beam strength: how to model it, measure it and mitigate it for various scientific fields}

\author{
John R. Helliwell*¥
}

Science and Engineering Research Council (now Science and Technology Facilities Council), Daresbury Laboratory, Warrington WA4 4AD, United Kingdom. *Correspondence e-mail: john.helliwell@manchester.ac.uk

It was 1983 when I was a beamline scientist at the UK's SRS responsible for Protein Crystallography Stations 7.2 (bending magnet) and 9.6 (superconducting high field wiggler, 5 T) and I was on the European Synchrotron Radiation Project (ESRP) Working Group for Biology chaired by Dr Joan Bordas. We had just received the spectral emission specifications from the ESRP Project Leader Professor Bronislaw Buras based at CERN in Geneva. The spectral brightnesses and fluxes of the various ESRP sources, especially the undulators, showed colossal gains over what we had at the SRS. At SRS 9.6 I had already insisted to the engineers involved on having a straight-through beam option. The melting of lead by the white beam (Fig. 1) was a vivid effect to show us what we had at our disposal. It was one of the first ever synchrotron source wigglers. Duly concerned I measured the temperature rise in a sample of water in one of our standard protein crystal mounting glass capillaries, which (from memory) was about $1^{\circ} \mathrm{C}$. The details of my test were very similar to those given in Fig. 11 of Cheng \& Caffrey (1996) who used CHESS $13.6 \mathrm{keV} \mathrm{X}$-rays of $2 \times 10^{10}$ photons s${ }^{-1}$ into a beam of size $0.3 \mathrm{~mm}$ and who reported a $0.16 \mathrm{~K}$ temperature rise. Therefore beam heating even on a second-generation source wiggler was not to be an anxiety for us as facility provider.

But, the beam strengths from the first ever third-generation source were far more (by up to $\sim 1000$ more photons per second per $\mathrm{mm}^{2}$ into beam sizes of about $100 \mu \mathrm{m}$, instead of SRS 9.6 of $\sim 1 \times 10^{11} 12 \mathrm{keV}$ photons into a focus with our optics of $\sim 1 \mathrm{~mm} \times$ $0.5 \mathrm{~mm}$ ). Another major feature of the ESRP specifications was that this was a $5 \mathrm{GeV}$ ring (ESRF was implemented at $6 \mathrm{GeV}$ so that the undulator emission was better matched to the requested Mossbauer experiments). There were to be copious quantities of higher-energy photon fluxes to be available, i.e. we could consider quite easily working with $0.5 \AA$ X-ray wavelength instead of typically $0.9 \AA$ wavelength on SRS 9.6 and $1.488 \AA$ on SRS 7.2 (both instruments were fully tunable for the anomalous dispersionbased studies).

Daresbury despatched me to work with Roger Fourme at the LURE synchrotron to consider these machine specifications in detail, namely their potential and yet also the possible problematic barriers to exploitation. I slept in the LURE users' dormitory cabin which I had sole use of as it was a shutdown; I recall that eating in Orsay on a Sunday evening did not offer much by way of opportunity. Ultimately I presented our report (Helliwell \& Fourme, 1983) to Professor Buras at CERN. We were actually very optimistic for macromolecular crystallography at the future ESRF. The calculations (Helliwell, 1984) showed that, in the worst case, the adiabatic model for the sample beam heating was serious but manageable. Specifically we proposed a new sample mounting to effect the best possible heat conduction away from the protein crystal. This comprised a crystal attached by grease to a copper stalk and surrounded by helium (Fig. 2). The grease would also protect the crystal from dehydration. Copper and helium were chosen for their high thermal conductivity. Later, at Daresbury and at other synchrotron sources we adopted the cryo-cooling methodology of Haas \& Rossmann (1970), encouraged especially by Hakon Hope and Ada Yonath for realizing successful ribosome crystallography measurements (Hope, 1988). Room-temperature diffraction measurements fell out of fashion and our crystal sample mounting protocol dimmed from view.

New third-generation sources steadily appeared, as have a succession of lower emittance, higher spectral brightness, machine upgrades. After 17 years a new sample heating 


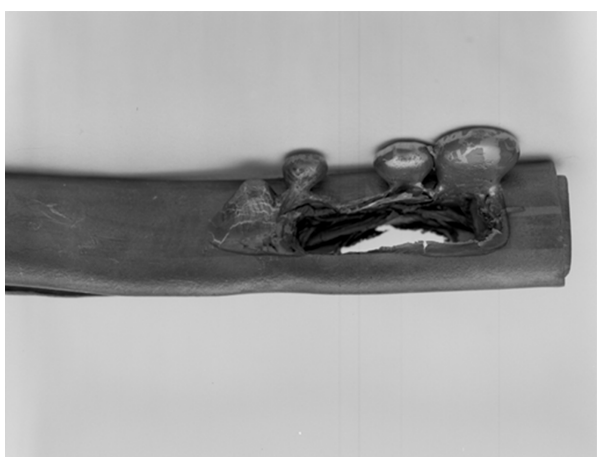

Figure 1

A piece of lead, aligned horizontally, at the SRS 9.6 melts due to the white beam.

publication appeared (Kuzay et al., 2001) and they even gave me a mention in their Contents' page Synopsis, although not quite how I would appreciate it:

'Improved thermal models that include convection are developed which replace Helliwell's adiabatic approximation. Temperature rises of $6 \mathrm{~K}$ are calculated for a cryocooled 100 micron thick crystal and of $18 \mathrm{~K}$ for a room-temperature air-cooled $1 \mathrm{~mm}$ thick crystal for an $8 \mathrm{keV} 10^{13}$ photons $\mathrm{s}^{-1} \mathrm{~mm}^{-2}$ beam. The importance of internal heat conduction within the crystal is also carefully examined.'

Apparently I was to be replaced rather than built upon.

I presented the different aspects of the Helliwell \& Fourme (1983) ESRP internal report in the journals' literature firstly in Helliwell (1984). The beam heating calculations had of course provided a basic 'worst case' adiabatic model. Kuzay et al. (2001) initiated the modelling of heat transfer from the sample, what I called an isothermal (i.e. steady state) model. We had of course immediately proposed an experimental solution, as mentioned above (Fig. 2). Further modelling studies, and measurements, followed [such as Snell et al. (2007)]. All these, including Kuzay et al. (2001), restricted themselves to modelling the case of a cryocooled protein crystal sample. An important exception was the extensive studies by Cherezov et al. (2002) who were constrained in their

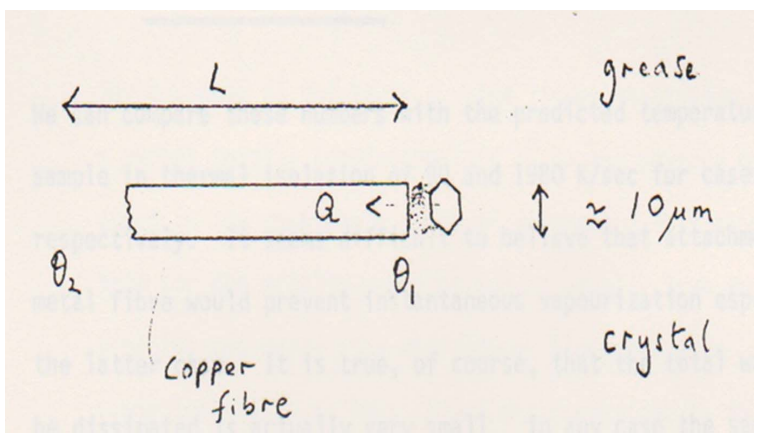

Figure 2

A strategy to control crystal sample heating was offered for roomtemperature X-ray diffraction measurements at the exceptionally large ESRF X-ray intensities that were planned (Helliwell \& Fourme, 1983). structural studies of lipid membranes and mesophases to room temperature.

Lawrence Bright et al. (2021) now lead the way for considering materials science and their samples' beam heating. They provide as context for their initiative a full citing of the macromolecular crystallography beam heating papers, to my knowledge [except the Cherezov et al. (2002) studies] and a selection of the radiation damage papers. This latter is a much bigger literature thanks to the major efforts of the International Symposia on Radiation Damage in Macromolecular Crystallography [see, for example, Garman \& Weik (2015)]. As part of the proposed ESRP and proposed New Rings at the Stanford Synchrotron Radiation Laboratory initiative led by Professors Keith Hodgson and Herman Winick, we also made evaluations of overall and specific (disulfide bond breaking) radiation damage (Hedman et al., 1985; Helliwell, 1988).

The context for Lawrence Bright et al. (2021) is that the ESRF's Extremely Bright Source (EBS) upgrade has been completed and even greater spectral source brightnesses duly realized at the turn of the year. They certainly respect their new ESRF EBS synchrotron beam strength. They offer their own ways of how to model it and measure it with different test samples relevant to materials science. They also offer ways to mitigate it. More than that, they suggest ways for experimenters to validate their measurements, learning from those experimental situations where experimenters should be concerned. As for the macromolecular crystallographers, the paper of Halle (2004) on cryo structural artefacts is encouraging us to more firmly establish when those artefacts might be serious. Our proposed sample mount (Fig. 2) may yet come into fashion. Also the helium gas would be in an enclosure allowing control of the crystal's temperature to be at $37^{\circ} \mathrm{C}$, i.e. physiological for mammals. That temperature would not allow much headroom as the protein melting temperature for mammalian proteins is around $60^{\circ} \mathrm{C}$. The alternative solution to Fig. 2, imported from the XFEL facilities, of serial crystallography of course implicitly assumes that every sample is identical. In a recent study I was thwarted in my detailed analyses because I merged the cryodiffraction data of two apparently identical crystals; I only made headway when I analysed them separately (Govada et al., 2021). Meanwhile, cryocrystallography continues to prove very effective in synchrotron facility measurement pipelines and with more and more remote working, as well as mitigating beam heating. These cryo crystal structures, the vast majority in the Protein Data Bank, have also dominated the training set though for prediction of 3D structures using deep learning from amino acid sequences (Helliwell, 2020).

Finally, I note that in Lawrence Bright et al. (2021) the adiabatic approximation has enjoyed a revival with six detailed mentions, and with much more besides. Suffice to say, Lawrence Bright et al. (2021) is I think an important paper.

\section{References}

Cheng, A. \& Caffrey, M. (1996). Biophys. J. 70, 2212-2222.

Cherezov, V., Riedl, K. M. \& Caffrey, M. (2002). J. Synchrotron Rad. 9, 333-341. 


\section{scientific commentaries}

Garman, E. F. \& Weik, M. (2015). J. Synchrotron Rad. 22, 195-200.

Govada, L., Saridakis, E., Kassen, S. C., Bin-Ramzi, A., Morgan, R. M., Chain, B., Helliwell, J. R. \& Chayen, N. E. (2021). IUCrJ, 8, 678-683.

Haas, D. J. \& Rossmann, M. G. (1970). Acta Cryst. B26, 9981004.

Halle, B. (2004). Proc. Natl Acad. Sci. USA, 101, 4793-4798.

Hedman, B., Hodgson, K. O., Helliwell, J. R., Liddington, R. \& Papiz, M. Z. (1985). Proc. Natl Acad. Sci. USA, 82, 7604-7607.

Helliwell, J. R. (1984). Rep. Prog. Phys. 47, 1403-1497.

Helliwell, J. R. (1988). J. Cryst. Growth, 90, 259-272.
Helliwell, J. R. (2020). IUCr Newsl. 28(4), 6.

Helliwell, J. R. \& Fourme, R. (1983). The ESRF as a facility for protein crystallography: A report and design study. ESRP Report IRI-4/83, pp. 1-36.

Hope, H. (1988). Acta Cryst. B44, 22-26.

Kuzay, T. M., Kazmierczak, M. \& Hsieh, B. J. (2001). Acta Cryst. D57, 69-81.

Lawrence Bright, E., Giacobbe, C. \& Wright, J. P. (2021). J. Synchrotron Rad. 28, 1377-1385.

Snell, E. H., Bellamy, H. D., Rosenbaum, G. \& van der Woerd, M. J. (2007). J. Synchrotron Rad. 14, 109-115. 